\title{
LAS CIRCUNSTANCIAS DE LA TOLERANCIA
}

\begin{abstract}
pesar de que todo el mundo habla de la «tolerancia», parece que no siempre se dice lo mismo cuando se usa esta palabra. Así, por ejemplo, Karl Raimund Popper afirma que la utopía democrática que anhela es una «utopía de la tolerancia» ${ }^{1} \quad$ y Robert Wolff define la tolerancia como «la virtud de la moderna democracia pluralista» ${ }^{2}$, en cambio, G. K. Chesterton ve en ella la virtud «de la gente que no cree en nada» ${ }^{3}$. Según Richard Vernon y Samuel LaSelva, tolerancia es un concepto político, pero, en modo alguno, una virtud ${ }^{4}$. A su vez, Peter P. Nicholson ve en la tolerancia un ideal moral ${ }^{5}$; Maurice Cranston, por su parte, la considera como «lo segundo mejor que debe ser apreciado en un mundo imperfecto» ${ }^{6}$. Finalmente, Herbert Marcuse señala los rasgos represivos de la «pura tolerancia del sentido y el sinsentido» en la sociedad democrática ${ }^{7}$ y Joshua Halberstan hasta sostiene la concepción según la cual la «tolerancia genuina» sería «fácticamente imposible para cualquiera» ${ }^{8}$.
\end{abstract}

${ }^{1}$ Karl Raimund Popper, «Toleration and Intellectual Responsibility» en David Edwards, Susan Mendus (comps.), On Toleration, Oxford, 1987, pág. 34.

${ }^{2}$ Robert Paul Wolff, «Jenseits der Toleranz» en R. P. Wolff, B, Moore, H. Marcuse, Kritik der reinen Toleranz» Francfort del Meno, 1988, pág. 9.

${ }^{3}$ G. K. Chesterton, citado en: Richard Vernon, Samuel LaSelva. «Justifying Tolerance», Canadian Journal of Polilical Science, 1984, tomo XVII, pág. 4.

${ }^{4}$ Cfr. Richard Vernon, Samuel LaSelva. «Justifying Tolerance», en Canadian Journal of Political Science, 1984, págs. 3 s.

${ }^{5}$ Peter Nicholson, «Toleration as a Moral Ideal» en John Horton, Susan Mendus (comps.), Aspects of Toleration, Londres/Nueva York, 1985, págs. 158 ss.

${ }^{6}$ Maurice Cranston, «John Locke and the Case for Toleration» en David Edwards, Susan Mendus (comps.), On Toleration, Oxford, 1987, pág. 102.

${ }^{7}$ Herbert Marcuse, «Represive Toleranz» en R. P. Wolff, B. Moore, H. Marcuse, Kritik der reinen Toleranz, Francfort del Meno, 1988, pág. 105.

${ }^{8}$ Joshua Halberstam. «The Paradox of Tolerance» en The Philosophical Forum, Winter 1982/83, tomo XIV:2, pág. 190. 
Esta selección de diferentes manifestaciones sobre la tolerancia permite inferir que los distintos autores se diferencian no sólo por lo que respecta a la evaluación sino también a la definición de la tolerancia. Pero, la diversidad de interpretación indica también que, en el caso de la tolerancia, se trata de un concepto importante que merece ser analizado más de cerca. Por ello, resulta tanto más necesario procurar aclarar el significado de la palabra «tolerancia». Esto es lo que aquí me he propuesto. Partiendo de la definición de tolerancia propuesta por Peter P. Nicholson, deseo considerar ahora las circunstancias que deben darse para que pueda hablarse de tolerancia.

\section{LA DEFINICIÓN DE TOLERANCIA DE NICHOLSON}

Según Nicholson, estamos frente a un acto de tolerancia si y sólo si una persona $A$ omite, por determinadas razones, intervenir (nonrejection) en contra de la acción $Y$ de una persona $B$, a pesar de que $Y(B)$ lesiona (deviance) una convicción relevante (importance) $X$ de $A$ (disapproval) y $A$ tiende y puede (power) actuar en contra de $Y(B)$. En lo que sigue, la palabra «acción» será usada en el sentido de G. H. von Wright, es decir, de forma tal que «designe no sólo intervenciones productivas o preventivas en el curso del mundo sino también omisiones» ${ }^{9}$.

Un acto de tolerancia, es decir, la omisión de una intervención en contra de $Y(B)$ después de sopesar las razones en pro y en contra de la intervención, está precedido por un determinado motivo es decir, la lesión de una convicción $X(A)$ por parte de la acción $Y(B)$, que es el objeto del acto de tolerancia.

Las condiciones mencionadas tienen que darse a fin de que pueda hablarse de tolerancia. Pero, ¿qué significa ser tolerante? Hay que distinguir la tolerancia de la propiedad «ser tolerante» ya que es posible, sin mayor problema, practicar una vez la tolerancia sin por ello ser una persona tolerante. Mientras que el acto de tolerancia consiste en la omisión de una intervención, la propiedad «ser tolerante» requiere una tendencia a practicar la tolerancia cada vez que se dan determinadas circunstancias. Esta tendencia no puede ciertamente ser observada directamente pero sí, en cambio, las circunstancias que deben darse para que pueda realizarse un acto de tolerancia. Cuando una persona $A$, cada vez que se dan estas circunstancias, practica la tolerancia, se puede inferir que $A$ tiene una tendencia o disposición a practicar la tolerancia

${ }^{9}$ Georg Henrik von Wright, Handlung, Norm und Intention, Berlín/Nueva York, 1977, pág.83. 
y, por lo tanto, posee la propiedad de ser tolerante. Ser tolerante es, pues, una propiedad disposicional.

\section{LAS CIRCUNSTANCIAS DE LA TOLERANCIA}

Siguiendo el enfoque de Ernesto Garzón Valdés con respecto a la propiedad disposicional de determinados sistemas políticos de ser estables, se puede decir que

Una determinada persona $A$ es tolerante si y sólo si, bajo determinadas circunstancias $C$, presenta la disposición a omitir una intervención, es decir, a no prohibir $Y(B)^{10}$.

En lo que sigue, habré de analizar más de cerca estas circunstancias.

\section{1. C'- La lesión de una convicción $X(A)$}

Para que se pueda hablar de tolerancia, tiene que existir, por lo pronto, una convicción $X(A)$ que es lesionada al tolerarse $Y(B)$. Como afirma Joshua Halberstam, no toda convicción (o, mejor dicho, su lesión) es un candidato para la tolerancia. Parece que sólo puede hablarse de un acto de tolerancia si se produce una lesión de una convicción relevante, es decir, la lesión de una opinión que juega un papel importante en el sistema personal de valores y reglas de $\mathrm{A}^{11}$. Cuanto mayor sea la importancia de esta convicción, tanto mayor el grado de tolerancia ${ }^{12}$.

Pero, ¿cómo puede saberse si en el caso de $X(A)$ se trata de una convicción relevante? La importancia de una convicción puede medirse, por ejemplo, observando si el disgusto (o irritación), es decir, el rechazo que provoca su lesión es tan grande que existe una tendencia a responder con una intervención. Esto significa que se está dispuesto, debido a la lesión de $X(A)$ por una acción $Y$, a llevar a cabo una reacción desagradable para $B$, de forma tal que se prohíba $Y(B)$, se la limite o reduzca. Cuando una madre $A$, con una sonrisa en los labios, no obliga a su hijo $B$ a que tome la última cucharada de sopa porque éste dice que

${ }^{10}$ Cfr. Ernesto Garzón Valdés, Die Stabilität politischer Systeme. Analyse des Begriffs mit Fallbeispielen aus Lateinamerika, Friburgo/Munich, 1988, págs. 25 ss.

${ }^{11}$ Cfr. también Peter Nicholson, «Toleration as a Moral Ideal», 1985, pág. 160. Entre los «constituents» de la tolerancia, incluye «Importance: The subject of the desviation $[Y(B)]$ is not trivial». Quizás habría aquí que agregar que la acción $Y(B)$ no tiene que ser considerada como trivial, al menos por $A$, ya que viola una regla de su sistema personal de reglas.

${ }^{12}$ Joshua Halberstam, «The Paradox of Tolerance» en The Philosophical Forum, 1982/83, págs. $191 \mathrm{~s}$. 
se la reserva para el hada de la sopa, parece que no podría decirse que estamos aquí frente a un acto de tolerancia. Manifiestamente la convicción de la madre de que no existe ningún hada de la sopa no es lo suficientemente relevante como para provocar en ella la tendencia a obligar a su hijo a que termine la sopa. Con otras palabras: en el sistema de reglas de $A$, $X$ no es tan importante como para que $A$ sienta el deseo de lanzar una prohibición contra $Y(B)$.

Las convicciones $X(A)$, cuya violación es percibida como disgusto, ofensa o hasta amenaza y, por lo tanto, provoca rechazo, pueden proceder de diferentes niveles del sistema de reglas de $A$. Entre ellos, cabe mencionar los siguientes:

\subsubsection{Contenidos}

a) Mandatos de la estética: Mary Warnock, por ejemplo, se considera a sí misma una persona tolerante porque no interviene cuando «uno de los amigos de su hija» se le presenta «con sandalias y traje, o con bastón y saco de tweed», cosa que le disgusta enormemente ${ }^{13}$.

b) Convenciones sociales: Un invitado provoca, por lo general, desagrado cuando no se atiene a las costumbres habituales de la buena educación en la mesa y, por ejemplo, hace ruidos desagradables o realiza simultáneamente las acciones de comer y de hablar. Ello puede tener como consecuencia que el dueño de casa no vuelva a invitarlo.

c) Prejuicios: Según Allport, un prejuicio es «una antipatía basada en una generalización falsa e inflexible» ${ }^{14}$ que puede conducir a un rechazo fanático, como lo documenta la persecución de los judíos durante el Tercer Reich. Sin embargo, la frase: «Soy tolerante frente a los judíos» les parece directamente absurda a quienes no comparten este prejuicio. Uno podría sentirse tentado a vincular los prejuicios exclusivamente con aquellos casos extremos tales como el del fanatismo contra los judíos, y llegar a la conclusión de que los prejuicios no pueden ser tomados en cuenta como motivos para la tolerancia. Sin embargo, los prejuicios no son en modo alguno lamentables fenómenos excepcionales, sino parte constitutiva del pensamiento humano. Tal como explica Lippman en Public Opinion, el hombre tiene que simplificar la realidad mediante la construcción de un «pseudo-entorno» a fin de poder manejarse con ella ${ }^{15}$. En el caso de los prejuicios, a este mecanismo de protección se suma un elemento de hostilidad. Sin

${ }^{13}$ Mary Warnock, «The Limits of Toleration» en David Edwards, Susan Mendus (comps.), On Toleration, Oxford, 1987, pág. 125.

${ }^{14}$ Gordon W. Allport, The Nature of Prejudice, Reading, Mass., 1954, pág. 10.

${ }^{15}$ Walter Lippmann, Public Opinion, Nueva York, 1922, pág. 16. 
embargo, «generalización y hostilidad forman parte de las capacidades naturales y comunes de la mente humana ${ }^{16} \mathrm{y}$, por lo tanto, influyen en el pensamiento y en la acción de toda persona. La tolerancia consiste aquí en tomar conciencia de los mecanismos que subyacen a los prejuicios y dominar la hostilidad que de ellos resulta ${ }^{17}$.

d) Principios de la racionalidad medio-fin: Cuando una persona actúa en contra de los mandatos del «sentido común», ello puede provocar también rechazo. Tal puede ser la sensación que experimenta el marido $A$ cuando su mujer $B$ se pone a limpiar todas las ventanas porque se avecina la Pascua, a pesar de que, con igual certeza, se avecina una tormenta que -como es fácil de predecir- anulará o reducirá apreciablemente el resultado de la «operación limpieza».

e) Convicciones religiosas: El rechazo que puede provocar la violación de convicciones religiosas es el tema de la Carta sobre la tolerancia de John Locke. Frente a las convicciones religiosas de quienes profesaban otras creencias religiosas, los cristianos estaban dispuestos a dejar de lado los mandamientos centrales de su propia religión, castigar a quienes consideraban equivocados y, hasta con la tortura y la pena de muerte, ayudarlos a que encontraran el «recto» camino. Esta pretendida «benevolencia celestial» es considerada, con razón, como un buen ejemplo de intolerancia.

f) Convicciones morales: La violación de valores morales suele también conducir al rechazo, con tendencia a la intervención. Muchas personas desaprueban, por ejemplo, el consumo y la distribución de material pornográfico. Su convicción $X$ de que las acciones sexuales son de una naturaleza eminentemente privada y no pueden ser expuestas públicamente resulta lesionada con la libre disposición de material pornográfico. Por ello, propician que el Estado dicte las correspondientes prohibiciones a fin de impedir lo que ellos consideran como un factor de la desintegración moral de la sociedad.

\subsubsection{Tipos de rechazo}

La lesión de estos diferentes niveles del sistema de reglas provoca formas de rechazo también diferentes. En la literatura anglosajona se suele distinguir entre «disapproval»y «dislike»; la primera expresión designa un rechazo fundamentado en razones prudenciales o morales; la segunda, en cambio, un sentimiento de rechazo.

${ }^{16}$ Gordon W. Allport, The Nature of Prejudice, Reading, Mass., 1954, pág. 17.

${ }^{17}$ Cfr. también Bernard Crick, «Toleration» en Government and Opposition, 197 1, pág. 145: "In many cases toleration need not imply the absence of prejudice, but only its containment and limitation». 
Peter Nicholson, por ejemplo, considera que la «disapproval» por razones morales puede dar lugar a un acto de tolerancia ya que la tolerancia constituye para él un ideal moral. Y, así, afirma:

«[U]na definición del ideal moral tiene que excluir 'dislike'... [T] $]$ enemos que ver el ideal moral de la tolerancia sólo en términos de desaprobación, es decir, de la formulación de juicios y el sostener razones sobre las cuales sea posible el argumento moral. La tolerancia es una cuestión de elección moral y nuestros gustos e inclinaciones son irrelevantes. $\rangle^{18}$

Y, así, según Nicholson, la tolerancia, en tanto ideal moral, está definida por las razones morales que abogan en favor del rechazo de lo que se tolera. Por lo tanto, estaríamos frente a un acto de tolerancia sólo si se prescinde de una prohibición de $Y(B)$, a pesar de que habrían razones morales para una intervención. La limitación que Nicholson lleva a cabo con respecto al acto de rechazo que considera como «susceptible de tolerancia» no es una mera cuestión de definición. Más bien parece que Nicholson confunde los planos que presentan a la tolerancia como un ideal moral.

En efecto, la tolerancia, en tanto resultado de una «elección moral», significa que hay que realizar una ponderación entre dos fundamentaciones morales recíprocamente incompatibles para luego decidirse en contra de una prohibición -también moralmente fundada- de Y(B).

Pero, según Nicholson, es también posible omitir intervenciones en virtud de razones no morales. Así, indica, por ejemplo, que la tolerancia puede ser el resultado de un «cálculo prudencial» ${ }^{19}$ y ser practicada cuando los costos que resultarían de una prohibición son demasiado altos. Pero, cuando razones morales imponen una intervención, ella no puede ser omitida por razones prudenciales ya que las razones morales son las razones últimas. El rechazo moralmente

${ }^{18}$ Peter Nicholson, «Toleration as a Moral Ideal», 1985, pág. 160. Esta opinión es compartida también por Joseph Raz. En su definición de tolerancia establece que la intervención a la cual uno tiende tiene que ser una acción «which is in itself morally valuable». Pero, la justificación moral de la intervención se basa en las razones morales para el rechazo, que coinciden con el deseo de intervención. Cfr. Joseph Raz, «Autonomy, Toleration, and the Harm Principle», 1987, pág. 320. Cfr. también Bernard Crick, «Toleration». Government and Opposition, 1971, pág. 157: También según Crick una de las circunstancias de la tolerancia consiste en que «there is moral disapproval with whatever embodies the threat». Sin embargo, en otro pasaje, señala que tolera "smoking in lectures and seminars» (pág. 150). Se puede suponer que rechaza el fumar en sus cursos no por razones morales, sino porque le molesta. Aquí hay, manifiestamente, una contradicción.

${ }^{19}$ Benjamín R. Barber, citado en: Peter Nicholson. «Toleration as a Moral Ideal», 1985,pág. 164. 
fundamentado puede ser superado sólo por mejores razones morales en favor de la no intervención. Supongamos, por ejemplo, que la feminista liberal $A$ rechaza la vestimenta de las mujeres iraníes porque considera que el «Tschador» es expresión de la opresión de la mujer. Esta justificación moral de su rechazo puede ser dejada de lado sólo en virtud de un «mejor» argumento moral en favor de la no intervención, por ejemplo, el reconocimiento del derecho de cada cual a seguir, dentro de ciertos límites, los mandamientos de su propia religión.

Parece pues que la tolerancia, en tanto ideal moral, es una cuestión de las razones morales que abogan en favor de la omisión de la intervención contra $Y(B)$. Aquí es irrelevante el tipo de rechazo -que esté fundamentado subjetiva, irracional o moralmente- que subyace al acto de tolerancia. Uno podría imaginar, por ejemplo, el caso de un modisto que, cuando ve un «Tschador», siente un rechazo y un deseo de intervenir porque esta vestimenta hiere su sensibilidad estética. Aquí no se trata de un caso de "disapproval» sino de "dislike», ya que sobre cuestiones de gusto no es posible aducir ningún argumento moral. No obstante ello, el modisto puede superar su rechazo en virtud de la reflexión moral según la cual cada persona tiene derecho a practicar libremente su religión.

Por lo tanto, el tipo del rechazo es irrelevante cuando se quiere precisar el tipo de tolerancia ${ }^{20}$. Lo decisivo para la «capacidad de tolerancia» de un rechazo no es su tipo, sino tan sólo la tendencia a la intervención que documenta la persistencia del rechazo que es desplazado por otras razones.

Las consideraciones anteriores han mostrado que una de las circunstancias $C$ de la tolerancia se da cuando la lesión de una convicción $X(A)$ provoca un rechazo de una intensidad tal que surge el deseo de proceder en contra de esta lesión. Pero, esto significa también que la intervención debe constituir una auténtica alternativa de acción, es decir, que $A$, en principio, tiene que estar en condiciones de proceder en contra de la lesión de una convicción relevante $X(A)$, es decir, en contra de $Y(B)$.

${ }^{20}$ Cfr. Mary Warnock. «The Limits of Toleration», 1987, pág. 135: «[M] ost of us, understand the objection to the use of human embryoes for research better than we understand the objection to hanging out the washing on Sunday. But for the person who objects to the latter, the sentiments of outrage may be indistinguishable». 


\section{2. $C^{2}$ : La posibilidad de intervenir como una cuestión de competencia}

Por ello, Peter Nicholson, al igual que muchos otros autores, afirma que la disponibilidad de medios de poder para la eliminación de $Y(B)$ forma parte de las circunstancias de la tolerancia. «El tolerante tiene el poder de tratar de suprimir o prevenir (o, al menos, de oponerse u obstaculizar) lo que es tolerado. «A ha de poseer, pues, la competencia o facultad que le permite fácticamente intervenir en contra de $Y(B)$.

Esto presupone, por lo pronto, que $A$, en principio, puede influir en $Y(B)$. Desde luego, tal no es el caso cuando $Y(B)$ es una catástrofe natural que escapa totalmente al control de $A$. Tal como correctamente afirma Bernard Crick, «parece no tener sentido tolerar o no tolerar cosas que están totalmente fuera de nuestro control» ${ }^{21}$. Por ello, sólo tiene sentido decir que $A$ posee la competencia para prohibir $Y(B)$ si $Y$ es una acción que realiza un ser humano $B$.

La competencia de $A$ para proceder en contra de $Y(B)$ puede, por ejemplo, tener un fundamento jurídico. Tal como explica Robert Alexy ${ }^{22}$, las competencias jurídicas constituyen «posiciones» que pertenecen a los «derechos».

Según Alexy, entre las competencias de todo ciudadano se cuentan, por ejemplo, la creación de asociaciones, la celebración del matrimonio, la adquisición de la propiedad o las disposiciones de última voluntad (Alexy, 220). El ciudadano se convierte así en «legislador privado» (Alexy, 217).

Un empresario, por ejemplo, tiene, dentro de su empresa, determinadas competencias legislativas. Así, puede disponer que dentro de su fábrica no se fume. Si, no obstante ser un convencido no fumador, supera su rechazo y permite fumar, demuestra tolerancia. Pero, si insiste en su prohibición de fumar, sus empleados están obligados a abstenerse de fumar. Por ello, no tiene sentido decir que los empleados toleran la prohibición de fumar impuesta por su jefe; tienen que soportarla si es que desean conservar su puesto de trabajo.

La competencia en virtud de la cual $A$ tiene la posibilidad de proceder en contra de $Y(B)$, que ella rechaza, puede ser también de naturaleza moral. Supongamos que una madre $A$ no está de acuerdo con que su hija mayor de edad $B$ cohabite con su amigo [Y]. Jurídicamente no tiene ningún recurso para proceder en contra de la decisión de su

${ }^{21}$ Bernard Crick. «Toleration». Government and Opposition, 1971, pág. 146.

${ }^{22}$ Robert Alexy, Theorie der Grundrechte, Francfort del Meno, 1986, págs. 211-224. Todas las explicaciones que aquí se formulan acerca del concepto de competencia jurídica se basan en las reflexiones de Alexy. 
hija. Pero, si la hija confiere un gran valor al juicio moral de su madre, ésta posiblemente estará en condiciones de lograr que su hija abandone sus planes [Y]. Si la madre, a pesar de que $Y(B)$ provoca en ella rechazo, se abstiene de ejercer su influencia moral, puede decirse que se muestra tolerante. En cambio, la hija no tolera las convicciones morales de su madre; si se abstiene de cohabitar con su amigo actúa en contra de sus propios deseos porque se siente moralmente obligado a seguir los deseos de su madre.

La competencia puede expresarse también bajo la forma de presión social. Cuando $B$ muestra con orgullo a sus amigos un cuadro que acaba de adquirir, pero constata que a nadie le gusta, posiblemente se abstendrá de colgarlo en la sala a fin de librarse de la crítica general. Pero, si sus amigos, no obstante su rechazo, se abstienen de formular comentarios negativos, a fin de no privarlo a $B$ del placer de su nueva adquisición, se muestran entonces tolerantes.

La posibilidad de proceder en contra de $Y(B)$ puede también basarse en una competencia fáctica. Ella se da cuando $A$ es superior a $B$ por lo que respecta a sus medios de poder. Los países industriales occidentales pueden, por ejemplo, debido a su poder económico, ejercer una gran influencia en las decisiones políticas de países dependientes amenazándolos con suspender su ayuda al desarrollo. Cuando se abstienen de utilizar su superioridad económica como medio de presión, a pesar de que no están de acuerdo, por ejemplo, con el desarrollo de la política interna del país receptor, demuestran tolerancia.

Manifiestamente, sólo puede practicar la tolerancia quien está en condiciones de no ser tolerante. Así, pues, se puede hablar de tolerancia sólo si $A$ goza de la libertad de decidir entre imponer u omitir una prohibición contra $Y(B)$. En este sentido, me parece correcta la afirmación de Kurt Biedenkopf según la cual la «voluntariedad... es un elemento constitutivo de la tolerancia $»^{23}$. El carácter voluntario de la tolerancia implica, primero, como ya se ha mostrado, que, en principio, $A$ posee competencia para proceder en contra de $Y(B)$. Pero, segundo, el ejercicio de esta competencia tiene que estar jurídicamente permitido dentro del marco de las leyes generales de la correspondiente sociedad, es decir, dentro del sistema de reglas de esta sociedad. Por lo tanto, no parece tener sentido valorar como tolerante el comportamiento de $A$ cuando se abstiene de matar a un opositor $B$ a causa de su acción $Y$ (por ejemplo, el ejercicio de su religión), a pesar de que fácticamente podría

${ }^{23}$ Kurt Biedenkopf, «Toleranz und Demokratie» en Uwe Schultz (comp.), Toleranz. Die Krise der demokratischen Tugend und sechzehn Vorschläge zu ihrer überwindung, 1974, pág. 168. 
hacerlo. Pero, la competencia de $A$ encuentra su límite en las leyes generales no sólo cuando el ejercicio de la competencia está prohibido sino también cuando el objeto de la tolerancia $Y(B)$ está ordenado, prohibido o autorizado en el sistema de reglas de la sociedad.

\subsubsection{Los límites de las competencias de A: la posición de Y(B) en el sistema de reglas de la sociedad}

Una evaluación entre la intervención y la no intervención es sólo posible si $Y(B)$ está nada más y nada menos que tácitamente permitido en el sistema de reglas de la sociedad, es decir, se encuentra en la «zona marginal de la libertad», en el «ámbito, pues, en el que los titulares de los derechos pueden disponer de ellos» ${ }^{24}$, como lo formula correctamente Kurt Biedenkopf.

Así, por ejemplo, la acción $Y(B)$ no puede ser objeto de la tolerancia si está prohibida en el sistema de reglas de la sociedad. $A$, no fumador y propietario de una gasolinera, no se ve enfrentado con la alternativa de prohibir fumar en su establecimiento o de superar su rechazo y permitir que se fume en la estación de servicio. Más bien está obligado a prohibirle a sus empleados $[B]$ que fumen $[Y]$, ya que existe una prohibición legal de fumar cuando hay material combustible cerca. $Y(B)$ está, pues, prohibido en las gasolineras y como el propietario carece de competencia legislativa para derogar esta prohibición, no cabe hablar de no intervención, es decir, de tolerancia con respecto a $Y(B)$.

Pero, la acción rechazada no puede tampoco ser objeto de tolerancia cuando se trata de una acción que está ordenada. El acompañante $A$ de un conductor $B$ no practica tolerancia alguna cuando le permite a este último que se ajuste a las limitaciones de velocidad en la carretera, aun cuando esta forma de conducir «poco deportiva» de $B$ le provoque un gran rechazo. Como $Y(B)$ está ordenado, $A$ no se encuentra frente a la elección de presionar a $B$ para que conduzca más rápidamente o de abstenerse de hacerlo. Como $A$ no está en condiciones de derogar las limitaciones de velocidad, está obligado a no proceder contra $Y(B)$.

Por lo tanto, no tiene sentido hablar de tolerancia frente a $Y(B)$ si $Y(B)$ está ordenado o prohibido en el sistema de reglas de la sociedad. Esto vale también para todas las acciones $Y(B)$ que están expresamente permitidas. Como afirman Richard Vernon y Samuel LaSelva, «... [el] reconocimiento de derechos de cualquier tipo vuelve innecesaria la tolerancia. Si tener un derecho es tener una elección

${ }^{24}$ Ibídem, pág. 173. 
protegida, entonces los derechos y los argumentos de la tolerancia difieren, al menos en un aspecto fundamental: la justificación de la omisión frente a la desaprobación es central en el caso de la tolerancia; pero, en el caso de los derechos, no surge ninguna cuestión de interferencia, ni siquiera en el caso de desaprobación y, por lo tanto, no puede surgir ninguna cuestión de omisión o de su justificación $\rangle^{25}$.

En Alemania, el policía que no detiene a los participantes de una manifestación autorizada no es tolerante, aun cuando se trate de partidarios del partido de extrema derecha NPD que, en su opinión, niegan los principios básicos de la democracia y, por lo tanto, ponen en peligro la seguridad general, no es tolerante. Su competencia para llevar a cabo detenciones está limitada por la ley según la cual no está autorizado a detener manifestantes que son partidarios de una organización política permitida en Alemania. También los partidarios del NPD tienen, de acuerdo con el artículo 5, párrafo 1 de la Ley Fundamental, «el derecho a expresar y difundir su opinión oralmente, por escrito y a través de la imagen...» como así también, de acuerdo con el artículo 8, párrafo 1 de la Ley Fundamental, a «reunirse pacíficamente y sin armas». Cuando una acción $Y(B)$ está expresamente permitida no puede ser objeto de la tolerancia ya que aquí se está obligado a no intervenir.

Quiero ahora resumir brevemente las diversas condiciones que hasta aquí he considerado con respecto al objeto de la tolerancia $Y(B)$ :

\subsubsection{La naturaleza del objeto de la tolerancia $Y(B)$}

En el caso de $Y(B)$, se trata de una acción que, en el sistema de reglas de la sociedad, está tácitamente permitida, pero lesiona una convicción en el sistema personal de valores de $A$ de forma tal que $A$ tiende, dentro de las posibilidades de que dispone jurídicamente, a lanzar una prohibición contra $Y(B)$.

No se trata necesariamente de una forma de comportamiento mala o incorrecta o que es percibida como tal. Como observa correctamente Raz, también los «reversos» de las virtudes pueden provocar profundo rechazo y, por lo tanto, ser buenos candidatos para la tolerancia. $B$ se distingue, por ejemplo, porque suele analizar todos los aspectos de un problema antes de someterlos a una cuidadosa evaluación. Ciertamente cuando se trata de casos difíciles en los que es importante llegar a una decisión bien fundamentada, ésta es una virtud que puede ser de gran

${ }^{25}$ Richard Vernon, Samuel LaSelva, «Justifying Tolerance» en Canadian Journal of Political Science, 1984, pág. 13. 
utilidad. Pero, cuando se trata de planear qué ha de hacerse un sábado por la noche y $B$ se niega a tomar una decisión antes de conocer todas las posibilidades alternativas y de llevar a cabo un estudio a fondo, recurriendo a suposiciones sofisticadas y a perspectivas contrafácticas, esta propiedad -que en otras circunstancias puede ser una virtud- ha de provocar probablemente un profundo rechazo en quienes no están dispuestos a invertir las horas del sábado por la noche en meditar acerca de qué es lo mejor hacer justamente ese sábado por la noche.

Según Raz, «existen varias formas y estilos de vida que ejemplifican diferentes virtudes y que son incompatibles». Esta idea de la existencia simultánea de varias formas de vida virtuosas es lo que él llama «pluralismo moral». Y es justamente este pluralismo moral lo que fatalmente contiene un potencial de intolerancia, porque el representante de una determinada forma de vida rechaza el comportamiento de las personas que siguen otra forma de vida, no por las virtudes que tienen, sino por la ausencia de las virtudes que no tienen porque son incompatibles con la respectiva forma de vida ${ }^{26}$.

\subsubsection{Conceptos afines}

Como se ha visto, las circunstancias de la tolerancia consisten en: a) el rechazo de una acción $Y(B)$ que va acompañado de la tendencia a prohibir $Y(B)$, estando b) jurídicamente permitido el ejercicio de la correspondiente competencia, y c) la omisión de la intervención, después de haber sopesado las razones en favor y en contra de la intervención. Quiero, por último, mencionar brevemente algunos conceptos que presentan una cierta afinidad con el de tolerancia. Ellos tienen en común con la tolerancia la circunstancia c), es decir, la omisión de la intervención, pero se distinguen de la tolerancia porque no se dan las circunstancias a) y/o b).

Como ya se ha insinuado, la tolerancia debe ser distinguida de la indiferencia. En la indiferencia, no se da la circunstancia a), es decir, el rechazo de $Y(B)$. Por ello, $A$ no tienen ningún motivo para proceder en contra de $Y(B)$.

El escepticismo es un caso especial de indiferencia que, a menudo, es confundido con la tolerancia. Así, como ya mencioné al comienzo, Chesterton afirma que la tolerancia es «la virtud de la gente que no cree en nada ${ }^{27}$. Pero, si no se cree en nada, tampoco puede sentirse ninguna

${ }^{26}$ Joseph Raz, «Autonomy, Toleration and the Harm Principle», 1987, págs. $316 \mathrm{~s}$.

${ }^{27}$ G. K. Chesterton, citado en: Richard Vernon, Samuel LaSelva. «Justifying Tolerance» en Canadian Journal of Political Science, 1984, pág. 4. 
tendencia a intervenir frente a una acción $Y(B)$. El deseo de prohibir $Y(B)$ presupone, en efecto, la existencia de una convicción importante que se está dispuesto a defender. Por ello, la tolerancia no es tampoco expresión de una actitud relativista, según la cual no existe verdad alguna de forma tal que «que todo vale; nada es defendible» ${ }^{28}$. En este caso, la tolerancia sería simplemente un sinónimo de indiferencia en vista de la imposibilidad de conocer la verdad y, así, poder asumir una posición ${ }^{29}$.

Tampoco parece que tiene sentido calificar como acto de tolerancia la abstención de una intervención por resignación. La resignación es la consecuencia de la impotencia, la convicción de $A$ de que no puede hacer nada en contra de $Y(B) n$ ya que carece de la correspondiente competencia. La resignación se diferencia, pues, de la tolerancia en virtud de la circunstancia b) de la tolerancia, es decir, la posibilidad de proceder en contra de $Y(B)^{30}$.

En los casos de indiferencia, de escepticismo y de resignación, no se da, pues, alguna o algunas de las circunstancias constitutivas de la tolerancia. Pero, también hay que distinguir de la tolerancia los casos en los que se presentan todas las circunstancias de la tolerancia pero sólo condicionalmente.

Un caso tal es el de la paciencia. Tanto la tolerancia como la paciencia se refieren a acciones $Y(B)$ que provocan el rechazo de $A$. El paciente -al igual que el tolerante- está en condiciones de prohibir $Y(B)$, pero no hace uso de su competencia. Sin embargo, en el caso de

${ }^{28}$ Karl Popper rechaza esta posición en: «Toleration and Intelectual Responsibility» en David Edwards, Susan Mendus (comps.), On Toleration, 1987, pág. 25. Según su opinión, el hombre es falible porque su conocimiento es limitado. Pero, falibilidad no implica que no exista ninguna verdad. A través de la discusión racional de diferentes opiniones, es posible una aproximación a la verdad recurriendo al principio de falsabilidad.

${ }^{29}$ Prescindiendo del hecho de que -al menos de acuerdo con la comprensión de este concepto aquí elaborada- un acto de tolerancia está precedido siempre por la violación de una convicción relevante para $A$, la tolerancia, en el sentido de un principio vinculante, es decir, el de omitir la intervención contra $Y(B)$, no es conciliable con el relativismo. Tal como afirma Ruth Zimmerling: «estas posiciones, en la medida en que infieren, a partir de premisas relativistas, un principio de tolerancia universal frente a otras posiciones éticas, es decir, un principio ético no relativista, son lógicamente inconsistentes.» (Cfr. Ruth Zimmerling, «Necesidades básicas y relativismo moral» en Doxa $n^{\circ} 7$ [1990], pág. 36.)

${ }^{30}$ Cfr. también Kurt Sontheimer, «Das Allgemeine und das Akademische» en Uwe Schultz (comp.), Toleranz. Die Krise der demokratischen Tugend und sechzehn Vorschlüge zu iherer überwindung, 1974, pág. 56: «Por lo demás, tiene poco sentido hablar de tolerancia cuando no puedo hacer nada en contra del comportamiento y el pensamiento de otras personas»». 
la paciencia, la circunstancia a), el rechazo, no está vinculada con una tendencia a la intervención, ya que la paciencia se basa en la esperanza o en la certeza de que su objeto tiene una existencia transitoria. El paciente $A$ no se siente, pues, impulsado a impedir o limitar $Y(B)$, ya que parte del supuesto de que en un tiempo prudencial, $Y(B)$ habrá dejado de existir. Sólo cuando se le rompe «el hilo de la paciencia», es decir, cuando desaparece su actitud de expectativa porque constata que $B$ no modificará su acción $Y$, surge una tendencia a intervenir, a la que -según el uso, en el lenguaje ordinario, de la expresión «perder la paciencia»- se suele también ceder. La paciencia presenta pues todas las circunstancias de la tolerancia pero, la circunstancia a) se limita al rechazo, sin que exista la tendencia a intervenir.

$\mathrm{Al}$ igual que la paciencia, la «tolerancia gubernamental», tal como Iring Fetscher llama a las disposiciones arbitrarias de tolerancia ${ }^{31}$, presenta todas las circunstancias de la tolerancia; pero, a la omisión de la intervención no subyace ninguna evaluación de las razones en favor o en contra de la intervención. Uno podría imaginar, por ejemplo, que $A$ arroja una moneda al aire y resuelve que en caso de «cara» prohibirá $Y(B)$ y, si sale «cruz», no procederá en contra de $Y(B)$. Pero, si $A$ hace depender su decisión de tolerar $Y(B)$ de la casualidad, es decir, si existe la misma probabilidad de que permita o no permita $Y(B)$, no parece tener mucho sentido hablar de tolerancia.

Parece, pues, que estamos frente a un acto de tolerancia siempre que se den plenamente las circunstancias presentadas. Así entendida, la tolerancia es un concepto valorativamente neutro. Las frases: «La persona $A$ es tolerante» o «La acción $Z$ es expresión de tolerancia» no tienen, por lo pronto, ninguna implicación positiva o negativa. Una evaluación de $A$ o $Z$ sólo es posible si se expresan las razones por las cuales se practica esta tolerancia. Cuando un gobierno, por razones de política de seguridad, tolera ciertas minorías, la tolerancia es un instrumento político. Pero, si el objeto del gobierno es proteger la igualdad y la libertad de todos los miembros de la sociedad, entonces practica la tolerancia en el sentido de una virtud democrática. Si un gobierno tolera incondicionalmente ciertas acciones, invocando el «principio de tolerancia», aun cuando éstas causan daño a una parte de los ciudadanos, la tolerancia puede convertirse fácilmente en un medio de represión, tal como lo pusiera críticamente de manifiesto Herbert Marcuse.

Sin la exposición de las razones para la omisión de la intervención en contra de $Y(B)$, no se entiende la frase lapidaria de Peter Nicholson:

${ }^{31}$ Cfr. Iring Fetscher, Toleranz. Von der Unentbehrlichkeit einer kleinen Tugend für die Demokratie, Stuttgart, 1990, pág. 11. 
Sin la exposición de las razones para la omisión de la intervención en contra de $Y(B)$, no se entiende la frase lapidaria de Peter Nicholson: «la tolerancia es correcta y el tolerante es bueno» ${ }^{32}$.

Es verdad que la historia de la tolerancia se presenta, en general, como un desarrollo estrechamente vinculado al de una mayor vigencia de los derechos humanos y de los principios de la democracia. Pero, no hay que olvidar que lo que confiere valor moral a la tolerancia son las razones que se invocan para practicarla. No puedo formular aquí mayores consideraciones al respecto. Tan sólo me interesaba presentar un enfoque analítico del concepto de tolerancia y subrayar la importancia de sus circunstancias.

${ }^{32}$ Peter Nicholson, «Toleration as a Moral Ideal», 1985, pág. 160. 